\title{
Effects of sex hormones on bronchial reactivity during the menstrual cycle
}

\author{
Maria Matteis ${ }^{1 \dagger}$, Francesca Polverino ${ }^{2,4,5 \dagger}$, Giuseppe Spaziano ${ }^{1}$, Fiorentina Roviezzo ${ }^{3}$, Carlo Santoriello ${ }^{4}$, Nikol Sullo ${ }^{1}$, \\ Maria Rosaria Bucci ${ }^{3}$, Francesco Rossi ${ }^{1}$, Mario Polverino ${ }^{4}$, Caroline A Owen ${ }^{2,4}$ and Bruno D'Agostino ${ }^{{ }^{*}}$
}

\begin{abstract}
Background: Many asthmatic women complain of symptom exacerbations in particular periods, i.e. during pregnancy and menstrual cycles (perimenstrual asthma: PMA)". The goal of this study was to study the effect of the luteal and follicular phases of the menstrual cycle on bronchial reactivity (BR) in a group of asthmatic women.

Methods: For this purpose, 36 pre-menopausal women were enrolled and underwent testing for resting pulmonary function, measurement of the diffusing capacity of the lung for carbon monoxide (DLCO), and airway responsiveness to methacholine in the follicular and luteal phases of their menstrual cycles. We also measured plasma hormone levels and levels of cyclic adenosine monophosphate (CAMP; a mediator of bronchial smooth muscle contraction) and testosterone in induced sputum samples.
\end{abstract}

Results: Our study showed that about $30 \%$ of the asthmatic women had decreased $\mathrm{PC}_{20} \mathrm{FEV}_{1.0}$ in the follicular phase of menstrual cycle with a significant correlation between $\mathrm{PC}_{20} \mathrm{FEV}_{1.0}$ and serum testosterone levels. Moreover, marked increases in sputum testosterone levels (mean $=2.6$-fold increase) together with significant increases in sputum cAMP concentrations (mean $=3.6$-fold increases) were observed during the luteal phase of asthmatic patients, suggesting that testosterone contributes to the pathophysiology of PMA. We excluded the possibility that testosterone directly inhibits phosphodiesterase (PDE) activity as incubating PDE with testosterone in vitro did not reduce PDE catalytic activity.

Conclusions: In conclusion, our data show that $\mathrm{PC}_{20} \mathrm{FEV}_{1.0}$ was decreased in the follicular phase of the menstrual cycle in about 30\% of women and was associated with lower cAMP levels in sputum samples, which may contribute to bronchoconstriction. Our results also suggest a link between PMA and testosterone levels. However, whether these findings are of clinical significance in terms of the management of asthma or asthma worsening during the menstrual cycle needs further investigation.

Keywords: Perimenstrual asthma, Menstrual cycle, Testosterone, Phosphodiesterases, Cyclic AMP

\section{Background}

Asthma is a chronic inflammatory disorder of the airways [1] in which many cells and cellular elements play a role $[2,3]$. The incidence, severity, and prognosis of asthma can be affected by several factors, including the patient's age and sex. Epidemiological studies of both incidence and prevalence have reported a male predominance of asthma before puberty and a female predominance after puberty.

Sex differences exist in the risk, incidence, and pathogenesis of various lung diseases in humans [4]. Females

\footnotetext{
*Correspondence: bruno.dagostino@unina2.it

${ }^{\dagger}$ Equal contributors

'Department of Experimental Medicine-Section of Pharmacology, 2nd University of Naples, 80136 Naples, Italy

Full list of author information is available at the end of the article
}

typically are more susceptible and/or develop more severe asthma, chronic obstructive pulmonary disease, lung cancer, and other lung conditions. Accumulating epidemiological and experimental data suggest that sex hormones may be important physiological modulators in the lung, and the role of estrogens in asthma has received considerable attention in this regard.

It has been known for a long time that some female asthmatic patients experience an aggravation of asthma symptoms during the premenstrual or menstrual phases of their cycle. In particular, it has been hypothesized that hormonal fluctuations during the menstrual cycle play a significant role in the pathophysiology of asthma, resulting in periodic worsening of disease severity in

\section{Biomed Central}


adult females [5-11]. Terms such as (pre-) menstrual, circamenstrual, or perimenstrual asthma (PMA) have been used to describe this phenomenon. Various approaches have been adopted to investigate the hormonal hypothesis. However, the roles of gender and sex hormones in asthmatic women are complex and not completely understood. A more complete understanding of the activities of hormones in regulating asthma exacerbations could introduce new strategies for symptom management and decrease the disease burden associated with this phenomenon.

Therefore, the goal of this project was to study the effect of the menstrual cycle; specifically, the luteal and follicular phases and plasma sex hormone levels, on bronchial reactivity (BR) in a group of asthmatic women. In particular, sex hormone levels and mediators of bronchial smooth muscle contraction were evaluated.

\section{Methods}

\section{Human study}

\section{Study population}

To study the relationship between bronchial reactivity and sexual hormones in menstrual cycle, 56 asthmatic premenopausal women were recruited from the outpatient clinics of the Respiratory Department of Cava de' Tirreni Hospital. We recruited women suffering from allergic asthma and documented bronchial hyperreactivity. We excluded women with a history of lung diseases other than asthma, coronary artery disease, congestive heart failure, or cor pulmonale. After accounting for refusals to participate $(\mathrm{n}=11)$, inadequate information on asthma status $(\mathrm{n}=3)$, and non-participation for other reasons $(\mathrm{n}=4), 38$ women $(70.4 \%)$ were enrolled in the study. After enrollment, 2 women were excluded because of data missing. A total of 36 females, aged between 23 and 43 years-old (mean: $32.6 \pm$ SD 4.3 yrs-old) completed the study. Among them, 5 were mild smokers with a smoking history of $8.2 \pm$ 2.9 pack/years. No subjects were using oral or intrauterine contraceptives. Obesity, defined as a BMI $\geq 30 \mathrm{~kg} / \mathrm{m}^{2}$, was present $\left(<35 \mathrm{~kg} / \mathrm{m}^{2}\right)$ in 5 females. At the time of the study, none of the patients had experienced recent acute exacerbations, and all of them were clinically stable.

Twenty-six (72\%) of the subjects had intermittent asthma, due to pollen aeroallergens: six patients (17\%) were sensitized to one allergen, twelve patients (33\%) were sensitized to two allergens, and eight patients (22\%) to three allergens. The remaining ten (28\%) women had persistent asthma, mainly due to perennial aeroallergens: five of them (14\%) were sensitized to one allergen, and five (14\%) were sensitized to two allergens. The mean values of total serum IgE were $449.7( \pm 336.9) \mathrm{kU} / \mathrm{L}$ in the first group (patients with intermittent disease) and 934.2 $( \pm 765.8) \mathrm{kU} / \mathrm{L}$ in the second group (patients with persistent disease). There was no significant correlation between severity of asthma and RAST classes.
The local Ethics Committee "ASL Salerno" approved the study ( $\mathrm{n}^{\circ} 357 / \mathrm{CdE}$ of September 12, 2012) and all respondents provided informed consent before participation.

At the time of enrollment, women were interviewed and completed a standardized questionnaire that included questions about demographic factors, pregnancy history, health care utilization, smoking history, years of education completed, marital status, asthma history, activity limitations due to asthma, household exposures, asthma-related emergency visits, use of drugs to treat asthma, and other chronic conditions.

\section{Measurement of IgE mediated hypersensitivity}

The patients underwent intradermal skin testing for IgEmediated hypersensitivity. Allergy skin-prick tests were carried out using 14 common aeroallergen extracts (Lofarma, Italy): Dermatophagoides (D.) farinae, D. pteronyssinus, cat, dog, ragweed mix, grass mix (Timothy, June, Orchard), ash, beech, birch, hickory, oak, poplar, and the molds Aspergillus and Alternaria tenuis. Histamine $(1 \mathrm{mg} / \mathrm{mL})$ and saline $(0.9 \%)$ solutions were used as positive and negative controls, respectively. Diagnosis of asthma was made on the basis of a history of asthma and the presence of a positive radioallergosorbent test (RAST) for IgE antibodies (RAST-CAPFEIA, Pharmacia, Uppsala, Sweden). Only women in whom there was an agreement between the results of the skin prick tests and the results of RAST were selected for the study.

\section{Pulmonary function test}

Patients were asked to refrain from using short-acting ß2agonists or caffeine-containing beverages for $12 \mathrm{~h}$ prior to testing. All enrolled subjects underwent assessment of pulmonary function at rest and measurement of DLCO and airway responsiveness to methacholine as outlined below. These tests were done twice on every subject both during the follicular and luteal phases of the menstrual cycle.

\section{Spirometry}

Measurements were performed according to American Thoracic Society criteria [12]. Spirometric maneuvers were conducted in triplicate and the highest $\mathrm{FEV}_{1.0}$ and FVC values were used in subsequent analyses. Predicted normal values of $\mathrm{FEV}_{1.0}$ and FVC were derived from standard equations. SVC was obtained by a slow inspiration from maximal end expiratory lung volume, before flow-volume loop measurement.

\section{Lung diffusion}

Diffusion capacity for carbon monoxide (DLCO; transfer factor) was obtained by the single-breath method and adjusted for hemoglobin and alveolar volume to yield the diffusion coefficient (KCO). For this purpose, subjects inspired gas atmospheric air mixtures containing $0.3 \% \mathrm{CH}_{4}$ and $0.3 \% \mathrm{CO}$. 


\section{Cholinergic responsiveness}

Cholinergic responsiveness was evaluated by challenging subject with increasing concentrations of inhaled methacoline approved following consensus recommendations. The magnitude of the effect was expressed as percentage of change from the control value $\left(\% \Delta \mathrm{FEV}_{1}\right)$. Airway reactivity was measured by inhaling increasing concentrations of methacholine from a DeVilbis nebulizer with a breathsynchronized trigger. The initial concentration of $1 \mathrm{mg} / \mathrm{ml}$ was progressively doubled until the $\mathrm{FEV}_{1.0}$ fell by $\geq 20 \%$ from its original value, and the provocative concentration ( $\mathrm{PC}_{20}$ meth) required to achieve this end point was determined by linear interpolation. The concentrations of methacholine were doubled also above the usual maximum concentration of $25 \mathrm{mg} / \mathrm{ml}$ in order to obtain a fall of $\mathrm{FEV}_{1.0}$ greater than $20 \%$ from the basal value in all subjects. Concentrations of methacholine greater than $25 \mathrm{mg} / \mathrm{ml}$ were required in 16 challenges ( 6 in the follicular phase and 10 in the luteal phase): 2 women needed a concentration greater than the usual maximum only in the follicular phase, 6 women only in the luteal phase, and 4 women in both phases. Bronchodilatation with standard aerosols of albuterol was done in order to reverse cholinergic responsiveness.

\section{Hormonal assessments}

A venous blood sample was drawn from each participant between $8 \mathrm{AM}$ and $10 \mathrm{AM}$ and serum was obtained by centrifugation. Serum aliquots were then stored at $-80^{\circ} \mathrm{C}$ until analysis.

Prolactin, follicule-stimulating hormone (FSH), luteinizing hormone $(\mathrm{LH})$, testosterone $(\mathrm{T})$, progesterone (PRG) and 17 $\beta$-estradiol (E2) were measured in all samples using an electrochemiluminescence immunoassay (ElecsysSystem, Roche Diagnostics GmbH, Mannheim, Germany) according to the manufacturer's instructions.

\section{Collection and analysis of induced sputum}

Induced sputum was collected pre- and post-shift by inhalation of isotonic $(0.9 \%)$ saline aerosol, in both the follicular and the luteal phases of the menstrual cycle, placed at $4{ }^{\circ} \mathrm{C}$ and immediately sent to the laboratory. After centrifugation, aliquots of the supernatant fluids were stored at $-80^{\circ} \mathrm{C}$ until further analysis. Cyclic AMP (cAMP) and testosterone levels were measured using Enzyme Immunoassays (Cayman-EIA kit, Michigan, USA). Moreover, PDE activity was evaluated using a PDE assay kit (BPS Bioscience, San Diego, CA). The measurements (PFTs, PC20, and blood collection) were performed on day $1 \pm 3$ days of menstruation and $14 \pm 3$ days after the start of menstruation.

\section{In vitro study}

\section{PDE activity assay}

The ability of testosterone to modulate PDE activity was evaluated by a colorimetric cyclic nucleotide phosphodiesterase assay, according to manufacturer instructions. This assay measures PDE mediated cleavage of cyclic adenosine monophosphate (cAMP) yielding 5 ' adenosine monophosphate (5'AMP), which is further cleaved into its nucleosides and phosphate components. The phosphate generated was quantified by a colorimetric reaction with Biomol green. A non-specific PDE inhibitor, 3-isobutyl-1methylxanthine (IBMX), was included as positive control for inhibitor screening. Physiologically-relevant concentrations of testosterone detected in plasma samples in normal human female subjects $(0.3$ and $1 \mathrm{nM})$ were tested and compared to IBMX $(40 \mu \mathrm{M})$.

\section{Statistical analysis}

\section{Statistical analyses for the human study}

Group data are expressed as mean $( \pm S D)$. As our data are generated from small patient populations and these data that are not normally distributed, we used the MannWhitney U-test for comparison between non-parametric results. The Spearman's rank correlation coefficient test was used to examine the association between functional data and hormone levels. These analyses were performed using the statistical package SPSS 17.0.

\section{Statistical analyses for in-vitro evaluations}

We performed analyses with two-way ANOVA within groups, followed by the Bonferroni test to correct for multiple comparisons. Data are presented as means \pm SEM.

Statistical analyses were performed using GraphPad Prism version 4.7 (GraphPad, San Diego, CA).

Statistical significance was defined as $P<0.05$.

\section{Results}

\section{The human study}

Among the 36 study subjects studied, 23 had at least one pregnancy with a total of 31 births (with 14 male and 17 female offspring). In 1 out of 14 male births, mothers reported an overall deterioration in symptoms, and in 2 out of 14 male births, mothers reported an improvement in symptoms. However, in 2 out of 17 female births, mothers reported an overall deterioration in symptoms but no mothers of female offspring reported improvement in symptoms. Twelve out of 36 women complained occasionally of perimenstrual asthma, but no patient complained of perimenstrual asthma during the study.

\section{PFTs}

Pulmonary function tests showed the absence of restrictive or obstructive defects, as detected by normal total lung capacity with normal $\mathrm{FEV}_{1.0} / \mathrm{FVC}$ ratio and normal DLCO values in both the follicular and the luteal phases of the menstrual cycle in all study subjects. The mean values for $\mathrm{PC}_{20} \mathrm{FEV}_{1.0}$ were not significantly different when measured either in the follicular and the luteal phases (Table 1). 
Table 1 Mean values of pulmonary function tests performed on asthmatic women in the follicular and luteal phases of menstrual cycle

\begin{tabular}{lcccccc}
\hline & FVC & FEV $_{1.0}$ & PEF & FEF & DLCO & PC $20.0^{20}$ \\
& \% pred. & $\%$ pred. & $\%$ pred. & \% pred. & $\%$ pred. & mg/ml \\
\hline Follicular & $103 \pm 13$ & $94 \pm 15$ & $78 \pm 17$ & $72 \pm 33$ & $82 \pm 30$ & $21 \pm 46$ \\
Luteal & $97 \pm 28$ & $91 \pm 18$ & $76 \pm 21$ & $65 \pm 26$ & $81 \pm 22$ & $35 \pm 56$ \\
\hline P $>0,05$. & & & & & &
\end{tabular}

However, simple plots of $\mathrm{PC}_{20} \mathrm{FEV}_{1.0}$ values in both follicular and luteinic phases showed that 10 out of 36 (27.8\%) of the women had lower $\mathrm{PC}_{20} \mathrm{FEV}_{1.0}$ values during the follicular phase (Figure 1).

\section{Plasma hormones}

Table 2 shows mean values ( \pm 1 S.D.) of plasma hormones during the follicular and luteal phases, and Figures 2, 3 and 4 show the relationships between any hormone (independent variable) and $\mathrm{PC}_{20} \mathrm{FEV}_{1.0}$ (dependent variable). Statistically significant differences were obtained only when correlating $\mathrm{PC}_{20} \mathrm{FEV}_{1.0}$ and plasma testosterone levels suggesting that testosterone modulates BR. A stepwise regression analysis, in which all clinical, functional, and hormonal data from both phases of the menstrual cycle were included, identified plasma testosterone levels as the only independent variable (Figure 2A).

\section{Cyclic AMP (cAMP) levels, testosterone levels and PDE activity in induced sputum}

Cyclic AMP is a key intracellular mediator that contributes to bronchoconstriction in asthmatic airways. Accordingly, we evaluated whether cAMP is present in asthmatic sputum samples and correlates with different phases of the menstrual cycle. As shown in Figure 5, sputum cAMP levels were significantly higher in the luteal phase when compared with the follicular phase of the menstrual cycle.

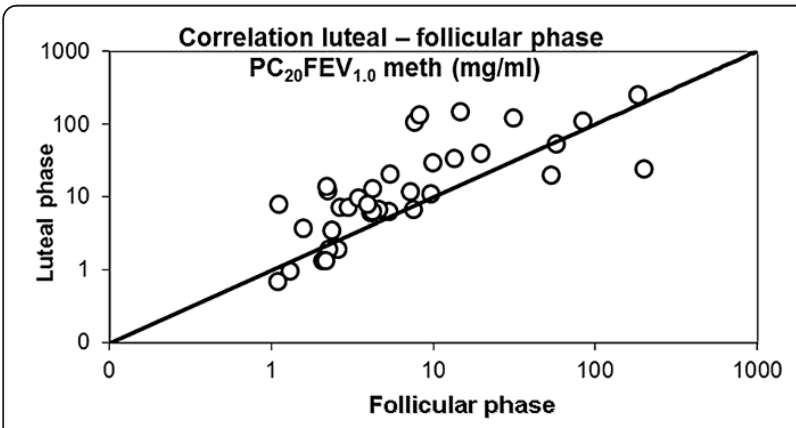

Figure 1 Correlation between the provocative concentration of methacoline ( $\mathrm{PC}_{20}$ meth) required to obtain a reduction in $\mathrm{FEV}_{1.0} \geq 20 \%$ from the baseline value. On the $x$-axis: $P C_{20}$ meth values measured in the follicular phase; on the $y$-axis: $\mathrm{PC}_{20}$ meth values measured in the luteal phase. About $30 \%$ of the women had lower $\mathrm{PC}_{20} \mathrm{FEV}_{1.0}$ values during the follicular phase (and vice versa). A logarithmic scale is used on both axes.
Table 2 Mean values ( \pm 1 S.D) of plasma hormones during the follicular and luteal phases

\begin{tabular}{lcccccc}
\hline & $\begin{array}{c}\text { FSH } \\
\mathbf{m U l} / \mathbf{m L}\end{array}$ & $\begin{array}{c}\mathrm{ng} / \mathbf{m L} \\
\text { Follicular }\end{array}$ & $\begin{array}{c}\text { TST } \\
\mathbf{n g} / \mathbf{m l}\end{array}$ & $\begin{array}{c}\text { LH } \\
\mathbf{m U l} / \mathbf{m L}\end{array}$ & $\begin{array}{c}\text { ESTR } \\
\mathbf{p g} / \mathbf{m L}\end{array}$ & $\begin{array}{c}\text { PRG } \\
\mathbf{n g} / \mathbf{m L}\end{array}$ \\
\hline Luteal & $12+8$ & $8 \pm 3$ & $0,33 \pm 0,09$ & $21 \pm 12$ & $47 \pm 20$ & $1,46 \pm 1,57$ \\
\end{tabular}

Interestingly, measurement of testosterone levels in the same samples revealed a similar profile, with significantly higher sputum levels of testosterone in the luteal phase compared with the follicular phase of menstrual cycle (Figure 6). Cyclic AMP levels are regulated by phosphodiesterases, which hydrolyze and thereby, reduce cAMP levels in cells. Thus, we measured PDE activity in sputum samples from the our asthmatic patients. Sputum PDE activity showed a different trend when compared with to those of cAMP and testosterone, as they were lower in the luteal phase when compared with sputum PDE activity levels measured in the follicular phase of menstrual cycle (Figure 7).

\section{In vitro study}

\section{Testosterone does not reduce PDE activity in vitro}

Next, we assessed whether testosterone directly reduces PDE activity to thereby increase cAMP levels in airway samples from patients with asthma. To assess this possibility, we incubated cell-free, purified PDE with or without two different concentrations of testosterone in vitro, and measured residual PDE activity. As shown in Figure 8, testosterone had no direct effect on PDE activity at either concentration tested. Thus, it is unlikely that testosterone modulates cAMP levels in asthmatic airways by directly interfering with PDE activity.

\section{Discussion}

Perimenstrual worsening of asthma with an increase in symptoms and significant decline in peak flow (20\%) has been reported in $30-40 \%$ of women [13]. Our study, carried out on 36 asthmatic women, has documented that 10 out of $36(27.8 \%)$ of them showed a trend towards a lower $\mathrm{PC}_{20} \mathrm{FEV}_{1.0}$ in the follicular phase when compared with their response in the luteal phase (or vice versa). However, this result was not statistically significant due to the high degree of variability between subjects, and was not clinically evident in terms of asthma-related symptoms. In this study, we have also demonstrated that in the luteal phase of menstrual cycle the mean level of testosterone in both sera and induced sputum samples is increased when compared with mean levels measured in the follicular phase. Higher testosterone levels in sputum samples were, in turn, associated with higher cAMP levels and lower PDE activity in sputum samples during 

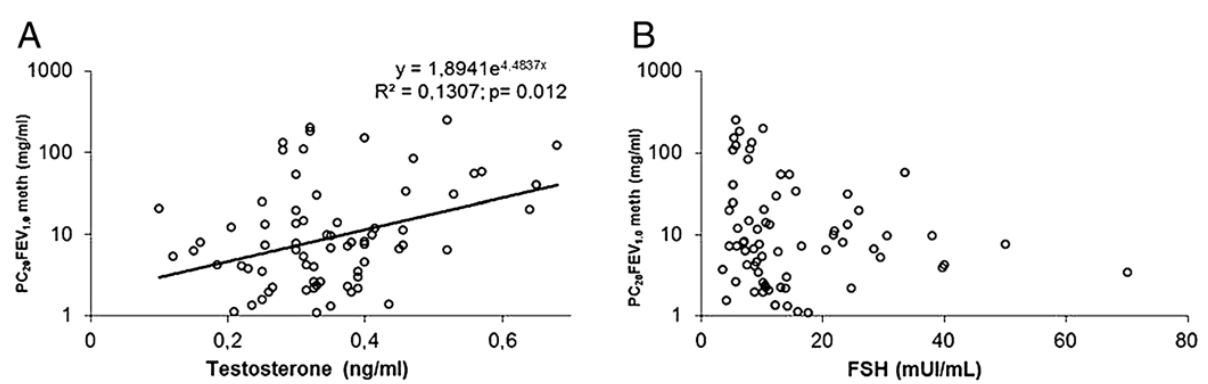

Figure 2 Relationship in each subject, measured twice (in both the follicular and luteal phases), between serum testosterone (panel A) and $\mathrm{FSH}$ (panel $\mathrm{B}$ ) on the $\mathrm{x}$-axes versus the $\mathrm{PC}_{20}$ meth using a logarithmic scale on the $\mathbf{y}$-axes. Only the relationship between testosterone and $P C_{20}$ meth reached statistical significance. $P<0.05$.

the luteal phase suggesting that testosterone regulates the generation of cAMP by reducing PDE activity.

A few studies have focused on bronchial hyperresponsiveness to various constrictor agents during the menstrual cycle in asthmatic women [8,9,13-18], but no cyclical variations were found in the subjects' responses to methacholine or histamine challenges $[8,9,13,14]$. However, two studies by Tan et al. $[15,16]$ showed an increase in $\mathrm{PC}_{20}$ in response to adenosine monophosphate challenges in the luteal phase when compared with responses in the follicular phase. This could indicate an effect of female sex hormones on airway inflammation during the menstrual cycle, as changes in AMP closely reflect changes in eosinophilic airway inflammation [17-19].

Women of reproductive age experience cyclic variations in serum concentrations of sex hormones [20]. During the 4 days after menstruation, FSH, LH, 17- $\beta$ estradiol, progesterone, and testosterone levels are low. During the follicular phase of the menstrual cycle, progesterone and testosterone serum levels remain low, while levels of FSH and LH peak. Finally, during the luteal phase, FSH and LH levels are low, whereas 17 - $\beta$-estradiol, progesterone, and testosterone are moderately high [20].
Clinical observations indicate that the hormonal fluctuations described above might be responsible for the cyclic changes in asthmatic symptoms reported by patients $[5,7,21,22]$. Our study adds changes in testosterone levels to the known hormonal fluctuations that have been demonstrated during the menstrual cycle. Our study documented a significant positive correlation between $\mathrm{PC}_{20} \mathrm{FEV}_{1.0}$ and serum testosterone levels during the follicular phase. In contrast, plasma levels of other sex hormones that we measured did not correlate with $\mathrm{PC}_{20} \mathrm{FEV}_{1.0}$. Moreover, we found significantly higher testosterone levels in induced sputum of our patients during their luteal phases when compared with testosterone levels measured in the follicular phases of their menstrual cycles.

Patients presenting premenstrual asthma worsening are frequently affected by alterations of the cyclic changes in serum levels of progesterone [20,23]. Progesterone, like all other steroid hormones, is synthesized from pregnenolone. Progesterone is the precursor of aldosterone, which in turn can be converted to testosterone [24]. It has been suggested that low testosterone levels may significantly alter immune responses and airway smooth muscle reactivity [25], through genomic or
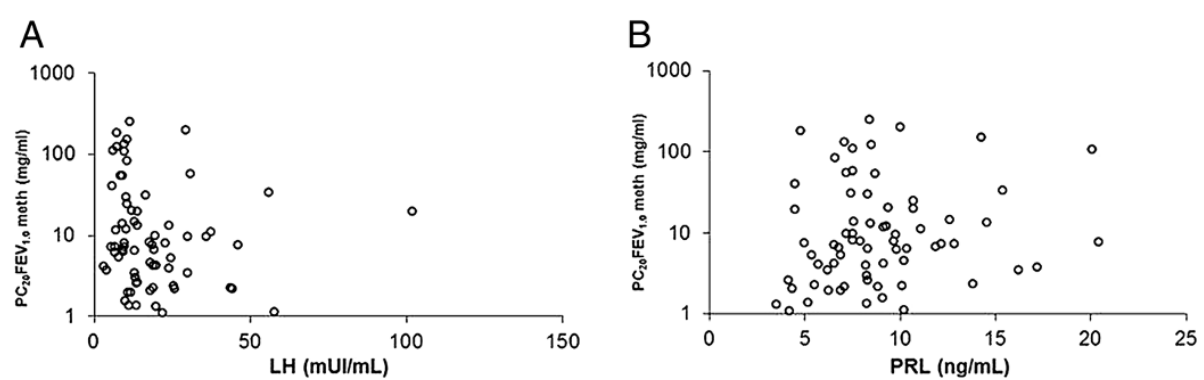

Figure 3 Relationship in each subject, evaluated twice (in both the follicular and luteal phases), between serum LH (panel A) and prolactin (PRL; panel B) levels on the $x$-axes versus the PC20meth on the $y$-axes using a logarithmic scale. No one of the relationships reached statistical significance. $P>0.05$. 

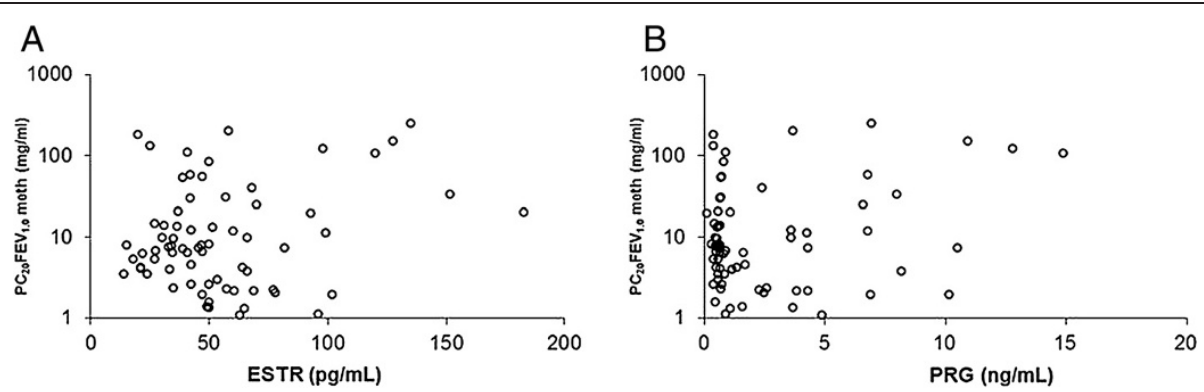

Figure 4 Relationship in each subject, evaluated twice (in both the follicular and luteal phases), between serum 17 $\beta$-estradiol (ESTR; panel A) and progesterone (PRG; panel B) levels on the x-axes versus the PC20meth on the y-axes using a in logarithmic scale. No one of the relationships reached statistical significance. $P>0.05$.

non-genomic mechanisms. Wulfsohn et al., treated female asthmatic patients with different hormones including testosterone and demonstrated an improvement in symptoms in $~ 90 \%$ of them showing an improvement in about $90 \%$ of them [26]. Furthermore, Mileva et al., found low levels of testosterone in blood samples from patients with severe and moderately severe asthma when compared with patients with mild asthma [27]. In our study, in addition to higher testosterone levels, we also documented significantly higher cAMP concentrations and lower PDE activity in induced sputum samples from patients in the luteal phase when compared with PDE activity measured during the follicular phase. PDEs are a diverse family of enzymes that play a key role in reducing levels of the second messenger, cAMP, and hence bronchial smooth muscle tone. Thus, the relatively low PDE activity in sputum samples from patients during the luteal phase of the menstrual cycle may contribute to the increased airway levels of cAMP

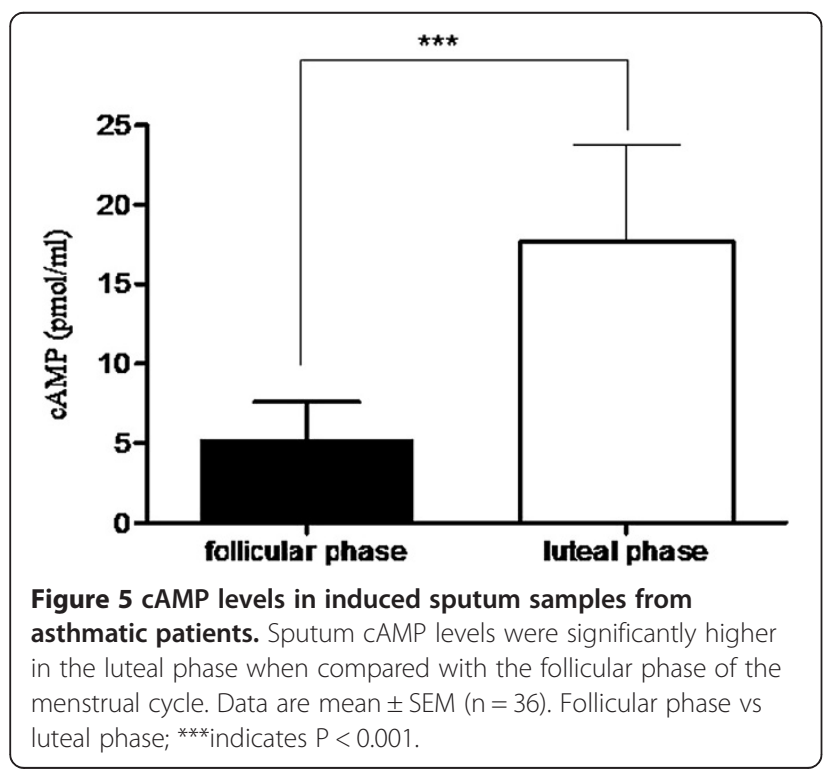

in these subjects in their luteal phase and vice versa for the follicular phase. Although sputum testosterone levels correlated indirectly with sputum PDE activity during the various phases of the menstrual cycle, our in vitro results exclude the possibility that testosterone directly inhibits the catalytic activity of PDE. It is possible that an as-yetunidentified testosterone-induced factor inhibits PDE activity in the airways of female asthmatics during the luteal phases of their menstrual cycles. In previous studies, we demonstrated that vasodilator effect of testosterone involves H2S, a novel gaseous mediator [28], which, in turn, may act as an endogenous inhibitor of PDE activity [29]. Therefore, we speculate that $\mathrm{H} 2 \mathrm{~S}$ mediates the inhibitory effect of testosterone on PDE activity in the airways of PMA subjects.

In rabbits, testosterone deficiency induced by castration reduced trabecular smooth muscle content, and this reduction was restored by testosterone treatment [30]. Moreover, the ability of testosterone to regulate PDE

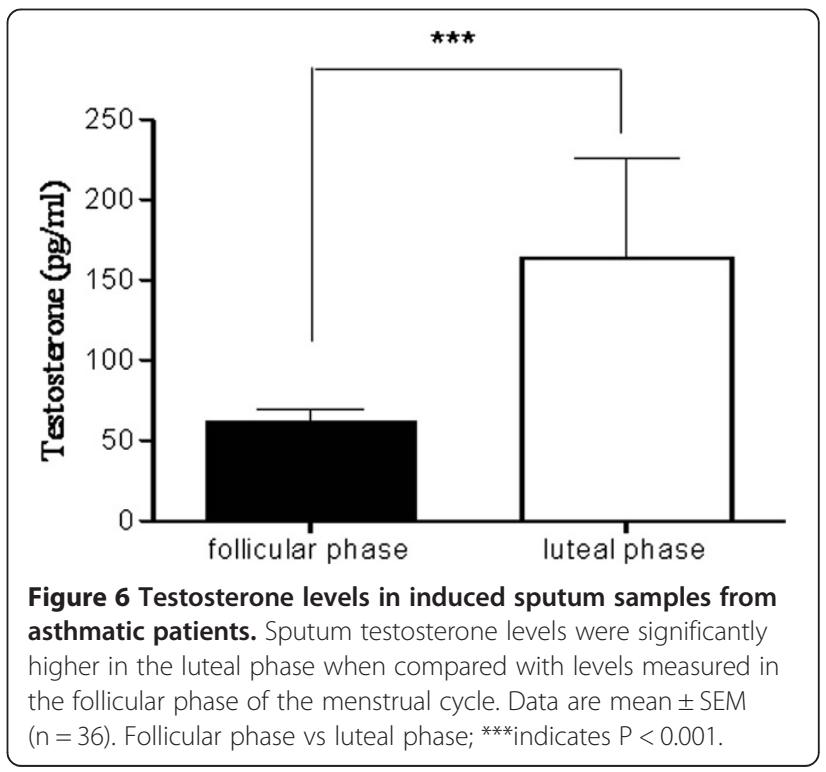




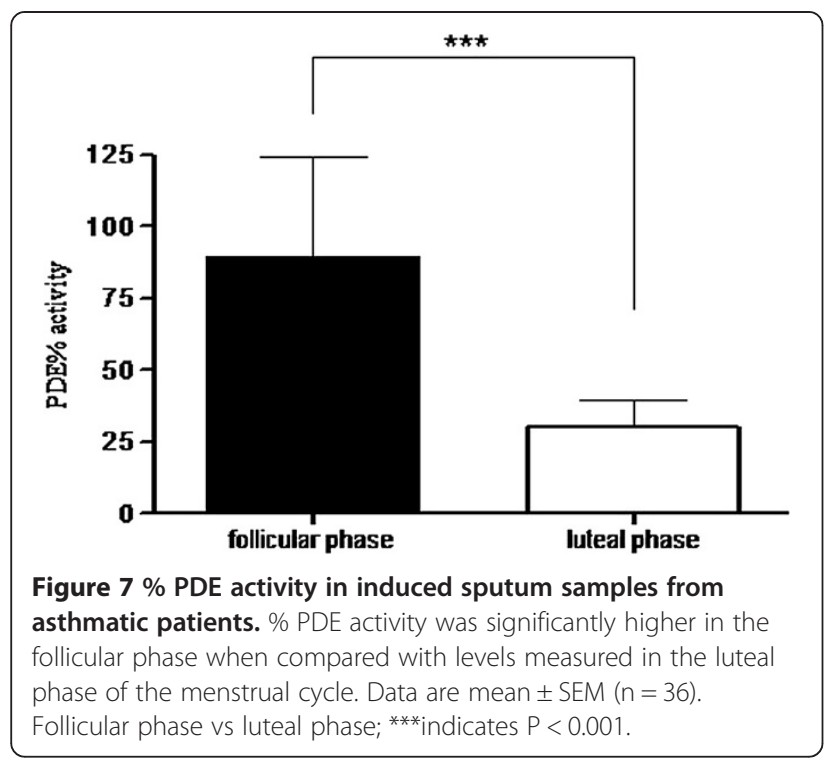

expression in rat corpus cavernosum has also been demonstrated [31]. In a human study, Aversa et al. investigated the role of androgens in regulating trabecular smooth muscle relaxation in the corpus cavernosum in men with erectile dysfunction in response to vasoactive challenge [32]. The findings indicated that, in men with erectile dysfunction, low testosterone levels correlate with impaired relaxation of cavernous endothelial and corporeal smooth muscle cells independently of age. These findings provide some clinical evidence that androgens regulate smooth muscle function in vivo. Finally, reduced responsiveness to $\beta 2$-agonists has been observed in surgically removed bronchial tissue from asthmatic

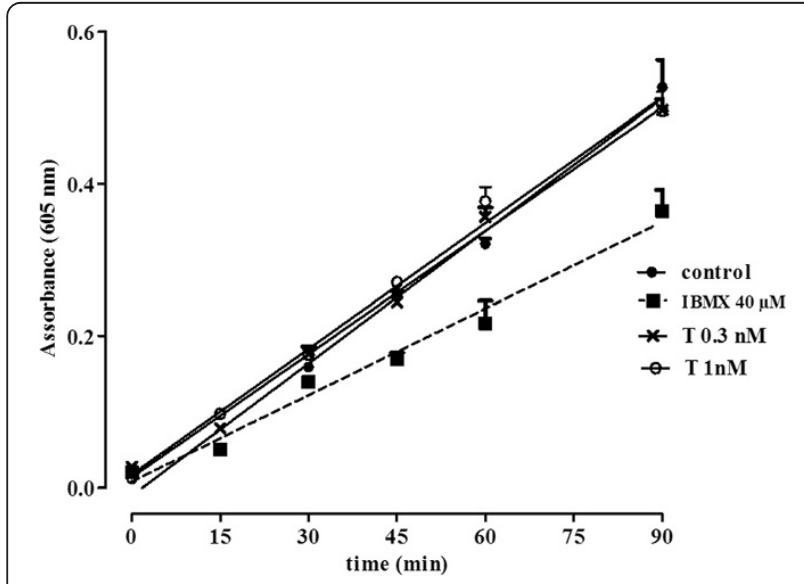

Figure 8 Testosterone does not affect PDE activity. PDE activity (cyclic nucleotide-degrading PDE activity) was evaluated in the presence of the indicated concentration of testosterone or IBMX, using a commercially available cyclic nucleotide phosphodiesterase kit. IBMX is a non selective PDEs inhibitor, used as positive control. $\mathrm{P}>0.05$. patients [33]. In addition, testosterone promotes smooth muscle relaxation in preparations of bronchial tissue isolated from asthmatic animals with desensitized betaadrenoceptors [34] and potentiates isoprenaline-mediated relaxation of bronchial smooth muscle [35]. Taken together, these studies suggest that smooth muscle tone in different tissues (trachea, bronchi, penis and the vasculature) is at least partially androgen-dependent.

The interpretation of our results should take into account the existing experimental and clinical data showing that female sex hormones influence lung function, airway responsiveness, and inflammation, but their effects vary depending on the experimental models and the end points analyzed [36].

\section{Conclusions}

In conclusion, our data provide evidence that variations in testosterone levels during the menstrual cycle may contribute to fluctuations of $\mathrm{PC}_{20} \mathrm{FEV}_{1.0}$ during the menstrual cycle of premenopausal women. Obviously, this is a biologic phenomenon that might not even have automatically clinical implication, as demonstrated by absence of perimenstrual asthma in our population during the study. Our results suggest that additional studies are needed to assess whether targeting testosterone and/or cAMP in asthmatic patients have potential to improve the management of the worsening of asthma during the menstrual cycle.

\section{Abbreviations}

PMA: Perimenstrual asthma; BR: Bronchial reactivity; DLCO: Diffusing capacity of the lung for carbon monoxide; CAMP: Cyclic adenosine monophosphate; PDE: Phosphodiesterase; FEV 1.0 : Forced expiratory volume in one second; FVC: Forced vital capacity.

\section{Competing interests}

The authors declare that they have no competing interests.

\section{Authors' contributions}

CS and MP performed the recruitment of patients and functional clinical study. GS, NS and MM performed assays of hormone levels in induced sputum and plasma. F Roviezzo and MRB performed the in vitro study of PDE activity. F Rossi and CAO made substantial contributions to the final version to be published. MM, FP, MP and BD made substantial contributions to conception, design and drafting the article. All authors read and approved the final manuscript.

\section{Acknowledgements}

We thank prof. Giuseppe Cirino from the Department of Experimental Pharmacology, University Federico II of Naples, for its substantial contributions to drafting the manuscript.

Supported by: MIUR PRIN 2009-2010 (RFr), MIUR PRIN 2010-2011 (BD).

\section{Author details}

'Department of Experimental Medicine-Section of Pharmacology, 2nd University of Naples, 80136 Naples, Italy. ${ }^{2}$ Division of Pulmonary and Critical Care Medicine, Brigham and Women's Hospital/Harvard Medical School, Boston, MA, USA. ${ }^{3}$ Department of Experimental Pharmacology, University Federico II of Naples, Naples, Italy. ${ }^{4}$ Cava de' Tirreni Hospital, Cava de' Tirreni, Italy. ${ }^{5}$ The Lovelace Respiratory Research Institute, Albuquerque, NM, USA. 
Received: 13 August 2013 Accepted: 26 June 2014

Published: 1 July 2014

\section{References}

1. Osman M: Therapeutic implications of sex differences in asthma and atopy. Arch Dis Child 2003, 88:587-590. Review.

2. Bateman ED, Hurd SS, Barnes PJ, Bousquet J, Drazen JM, FitzGerald M, Gibson P, Ohta K, O'Byrne P, Pedersen SE, Pizzichini E, Sullivan SD, Wenzel SE, Zar HJ: Global strategy for asthma management and prevention: GINA executive summary. Eur Respir J 2008, 31:143-178.

3. Singh SR, Sullo N, D'Agostino B, Brightling CE, Lambert DG: The effects of nociceptin peptide (N/OFQ)-receptor (NOP) system activation in the airways. Peptides 2013, 39:36-46.

4. Caracta CF: Gender differences in pulmonary disease. Mt Sinai J Med 2003, 70:215-224. Review.

5. Hanley SP: Asthma variation with menstruation. Br J Dis Chest 1981, 75:306-308

6. Eliasson O, Scherzer HH, DeGraff AC: Morbidity in asthma in relation to the menstrual cycle. J Allergy Clin Immunol 1986, 77:87-94.

7. Gibbs CJ, Coutts II, Lock R, Finnegan OC, White RJ: Premenstrual exacerbation of asthma. Thorax 1984, 39:833-836.

8. Pauli BD, Reid RL, Munt PW, Wigle RD, Forkert L: Influence of menstrual cycle on airway function in asthmatic and normal subjects. Am Rev Respir Dis 1989, 140:358-362

9. Juniper EF, Kline PA, Roberts RS, Hargreave FE, Daniel EE: Airway responsiveness to methacholine during the natural menstrual cycle and the effect of oral contraceptives. Am Rev Respir Dis 1987, 135:1039-1042.

10. Chandler MHH, Schuldheisz S, Phillips BA, Muse KN: Premenstrual asthma: the effect of estrogen on symptoms, pulmonary function, and B2-receptors. Pharmacotherapy 1997, 17:224-234.

11. Agrawal AK, Shah A: Menstrual-linked asthma. J Asthma 1997, 34:539-545.

12. Miller MR, Hankinson J, Brusasco V, Burgos F, Casaburi R, Coates A, Crapo R, Enright P, van der Grinten CP, Gustafsson P, Jensen R, Johnson DC, MacIntyre N, McKay R, Navajas D, Pedersen OF, Pellegrino R, Viegi G, Wanger J, J; ATS/ERS Task Force: Standardisation of Spirometry. Eur Resp J 2005, 26:319-338.

13. Shames RS, Heilbron DC, Janson SL, Kishiyama JL, Au DS, Adelman DC Clinical differences among women with and without self-reported perimenstrual asthma. Ann Allergy Asthma Immunol 1998, 81:65-72.

14. Weinmann GG, Zacur H, Fish JE: Absence of changes in airway responsiveness during the menstrual cycle. J Allergy Clin Immunol 1987, 79:634-638.

15. Tan KS, MCFarlane LC, Lipworth BJ: Modulation of airway reactivity and peak flow variability in asthmatics receiving the oral contraceptive pill. Am J Respir Crit Care Med 1997, 155:1273-1277.

16. Tan KS, MCFarlane LC, Lipworth BJ: Loss of normal cyclical beta 2 adrenoceptor regulation and increased premenstrual responsiveness to adenosine monophosphate in stable female asthmatic patients. Thorax 1997, 52:608-611.

17. van den Berge M, Kerstjens HA, Meijer RJ, de Reus DM, Koëter GH, Kauffman HF, Postma DS: Corticosteroid-induced improvement in the $\mathrm{PC}_{20}$ of adenosine monophosphate is more closely associated with reduction in airway inflammation than improvement in the $\mathrm{PC}_{20}$ of methacholine. Am J Respir Crit Care Med 2001, 164:1127-1132

18. Van den Berge M, Kerstjens HAM, Postma DS: Provocation with adenosine 50-monophosphate as a marker of inflammation in asthma, allergic rhinitis and chronic obstructive pulmonary disease. Clin Exp Allergy 2002, 32:824-830

19. Sullo N, Roviezzo F, Matteis M, lanaro A, Calò G, Guerrini R, De Gruttola L, Spaziano G, Cirino G, Rossi F, D'Agostino B: Nociceptin/orphanin FQ receptor activation decreases the airway hyperresponsiveness induced by allergen in sensitized mice. Am J Physiol Lung Cell Mol Physiol 2013, 304:657-664.

20. Erickson GF: Follicular growth and development. In Gynecology and obstetrics. Vol 5, chap 12. Edited by Lippincott. Philadelphia; 1991.

21. Settipane RA, Simon RA: Menstrual cycle and asthma. Ann Allergy 1989, 63:373-378.

22. Myers JR, Sherman CB: Should supplemental estrogens be used as steroid-sparing agents in asthmatic women? Chest 1994, 106:318-319.
23. Rubio RL, Rodriguez BG, Collazzo JJA, Heredia LB, Fernandez LF: Comparative study of progesterone, estradiol and cortisol concentrations in asthmatic and nonasthmatic women. Allergol Immunopathol (Madr) 1988, 16:263-266.

24. Nieschlag E, Behr H: Testosterone. Action, Deficiency, Substitution. 1st edition. Cambridge: Cambridge University Press; 2004.

25. Rogliani $\mathrm{P}$, Calzetta L, Rendina EA, Massullo D, Dauri M, Rinaldi B, Capuano A, Matera MG: The influence of propofol, remifentanil and lidocaine on the tone of human bronchial smooth muscle. Pulm Pharmacol Ther 2013, 26:325-331.

26. Wulfsohn NL, Politzer WM, Henrico JS: Testosterone therapy in bronchial asthma. S Afr Med J 1964, 38:170-172

27. Mileva Z, Maleeva A: The serum testosterone level of patients with bronchial asthma treated with corticosteroids and untreated. Vutr Boles 1988, 27:29-32.

28. Bucci M, Mirone V, Di Lorenzo A, Vellecco V, Roviezzo F, Brancaleone V, Ciro I, Cirino G: Hydrogen sulphide is involved in testosterone vascular effect. Eur Urol 2009, 56:378-383.

29. Bucci M, Papapetropoulos A, Vellecco V, Zhou Z, Pyriochou A, Roussos C, Roviezzo F, Brancaleone $V$, Cirino G: Hydrogen sulfide is an endogenous inhibitor of phosphodiesterase activity. Arterioscler Thromb Vasc Biol 2010, 30:1998-2004.

30. Traish AM, Park K, Dhir V, Kim NN, Moreland RB, Goldstein I: Effects of castration and androgen replacement on erectile function in a rabbit model. Endocrinology 1999, 140:1861-1868.

31. Zhang XH, Morelli A, Luconi M, Vignozzi L, Filippi S, Marini M, Vannelli GB, Mancina R, Forti G, Maggi M: Testosterone regulates PDE5 expression and in vivo responsiveness to tadalafil in rat corpus cavernosum. Eur Urol 2005, 47:409-416.

32. Aversa A, Isidori AM, De Martino MU, Caprio M, Fabbrini E, Rocchietti-March M, Frajese G, Fabbri A: Androgens and penile erection: evidence for a direct relationship between free testosterone and cavernous vasodilation in men with erectile dysfunction. Clin Endocrinol (Oxf) 2000, 53:517-522.

33. Fauci A, Braunwald E, Kasper D: Harrison's Principles of Internal Medicine. 17th edition. New York: McGraw Hill; 2008.

34. Suárez L, Pipa M, Granda J, Coto A, Bordallo J, Cantabrana B, de Boto García MJ, Sánchez M: Sex hormones modulate salbutamol-elicited long-term relaxation in isolated bovine tracheal strips. Pharmacology 2011, 87:249-256.

35. Forster PS, Goldie RG, Patterson JW: Effect of steroids on betaadrenoreceptor-mediated relaxation of pig bronchus. $\mathrm{Br} J$ Pharmacol 1983, 78:441-445.

36. McKenzie R, Burton MD, Royce SG, Tang M: Age and sex influences on airway hyperresponsiveness. J Asthma 2010, 47:651-654.

doi:10.1186/1471-2466-14-108

Cite this article as: Matteis et al.: Effects of sex hormones on bronchial reactivity during the menstrual cycle. BMC Pulmonary Medicine 2014 14:108.

\section{Submit your next manuscript to BioMed Central and take full advantage of:}

- Convenient online submission

- Thorough peer review

- No space constraints or color figure charges

- Immediate publication on acceptance

- Inclusion in PubMed, CAS, Scopus and Google Scholar

- Research which is freely available for redistribution 